\title{
Lifetime centric load balancing mechanism in wireless sensor network based IoT environment
}

\author{
Veerabadrappa ${ }^{1}$, Booma Poolan Marikannan ${ }^{2}$ \\ ${ }^{1}$ Department of Research and Development of a Product based USA Software Company, India \\ ${ }^{2}$ School of Computing, Asia Pacific University of Technology and Amp, Innovation, Malaysia
}

\begin{tabular}{l}
\hline \hline Article Info \\
\hline Article history: \\
Received Oct 11, 2020 \\
Revised Mar 14, 2021 \\
Accepted Mar 27, 2021
\end{tabular}

Keywords:

LCLBM

Lifetime maximization

Load balancing

Network

Wireless sensor

\begin{abstract}
Wireless sensor network (WSN) is a vital form of the underlying technology of the internet of things (IoT); WSN comprises several energy-constrained sensor nodes to monitor various physical parameters. Moreover, due to the energy constraint, load balancing plays a vital role considering the wireless sensor network as battery power. Although several clustering algorithms have been proposed for providing energy efficiency, there are chances of uneven load balancing and this causes the reduction in network lifetime as there exists inequality within the network. These scenarios occur due to the short lifetime of the cluster head. These cluster head $(\mathrm{CH})$ are prime responsible for all the activity as it is also responsible for intra-cluster and inter-cluster communications. In this research work, a mechanism named lifetime centric load balancing mechanism (LCLBM) is developed that focuses on $\mathrm{CH}$-selection, network design, and optimal $\mathrm{CH}$ distribution. Furthermore, under LCLBM, assistant cluster head (ACH) for balancing the load is developed. LCLBM is evaluated by considering the important metrics, such as energy consumption, communication overhead, number of failed nodes, and one-way delay. Further, evaluation is carried out by comparing with ES-Leach method, through the comparative analysis it is observed that the proposed model outperforms the existing model.
\end{abstract}

This is an open access article under the CC BY-SA license.

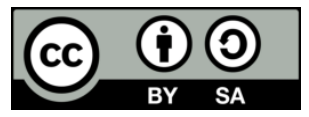

\author{
Corresponding Author: \\ Veerabadrappa \\ Department of Research and Development of a Product based USA Software Company \\ India \\ Email: veeru4998@gmail.com
}

\section{INTRODUCTION}

IoT can be potentially defined as the ecosystem of the interlinked devices through the internet for sending and receiving the data. It is one of the intelligent networks that can be controlled, sensed, and programmed through the embedded technology to share information [1]. Internet of things gives the earliest access to the devices performing with high efficiency and great productivity. Moreover, during the survey, it is found that nearly 5 billion devices are available in the world and the expansion of IoT suggests that by the end of 2020 this number will be 50 billion devices, not only that the number might also exceed in terms of several people communicating than the number of devices connected. These phenomena may cause huge traffic, this provides one of the multiple reasons to further research in the IoT area.

Internet of things has various applications, mainly e-health, retail and logistics, smart agriculture, smart home, and smart city. Nevertheless, these applications require some properties such as low delay, security, and reliability. The discussed characteristics are very important for the ideal utilization of IoT and it is observed that different applications require different characteristics. For instance, in the case of smart 
education and e-health; secure, reliable communication and private communication is required. Similarly, in case of an emergency solution, reliable communication and low delay are very important. Energy efficiency is required in every application; hence, load balanced communication must be developed in such a way that it can fulfill criteria [2]. Wireless sensor network (WSN) i.e., wireless sensor network acts as the bridge between the real world and digital world as the sensors are connected and they are responsible for sensing the data and transmitting the data through the internet [3]. WSN is nothing but the multiple sensors deployed in the network field for monitoring several parameters such as physical and environmental.

Figure 1 shows data transmission in the wireless sensor network. In Figure 1, there are many sensor nodes, and these nodes are clustered using the clustering algorithm [4], [5]. Here, the sensor nodes sense the data and send it to the corresponding cluster head, later these cluster head $(\mathrm{CH})$ transmits the data to a base station. Further, through the base station, data can be accessed by the internet and these sensors are batterybased, hence, the WSN model must be designed in such a way that it should be efficient in terms of selection of path, clustering, load balancing, sensing, and parameter design. WSN that is meant for IoT faces a lot of issues apart from sensing such as the amount of sensor node deployed in the field, hardware damage, communication mode, limited battery power, Increase in computational cost. Apart from these constraints WSN that is meant for IoT also faces the challenges of quality of service (QoS), power management, and security [6], [7]. Moreover, energy consumption has been a primal issue while designing the WSN model for IoT and this has led the researcher to get into depth for reducing the energy consumption and several ideas such as routing, and clustering have been put to reduce the energy consumption [8].

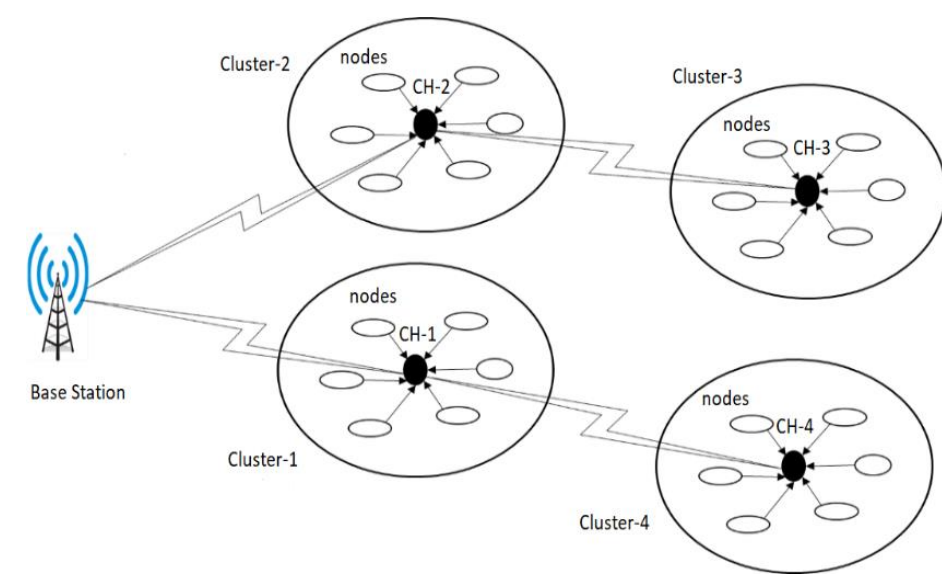

Figure 1. Data transmission in the wireless sensor network

a. Motivation and contribution of the research work

In WSN there are many clusters and each cluster have a cluster head and if one cluster head fails then the network becomes unbalance, and the data transmission fails. Other factors such as energy consumption cause a reduction in network lifetime [9]. Hence, energy depletion equilibrium or load balancing strategy was introduced to maximize the lifetime. In past, several methodologies have been proposed for load balancing [10], [11], however, these methodologies face issues such as network complexity, the lifetime of $\mathrm{CH}, \mathrm{CH}$-selection, clustering, and routing. Hence, motivated by the above discussion, this paper proposes a novel mechanism named lifetime centric load balancing mechanism (LCLBM) which focuses on maximizing the network lifetime. Further, the contribution of research work is highlighted through the below points:

The main aim of LCLBM is to maximize the network lifetime through balancing the load.

- Study and deep analysis of balanced clustering algorithm.

- At first, a design that is specific to network topology and energy model is developed, and later an adaptive mechanism of cluster head sharing is developed.

- Further, develop the novel $\mathrm{CH}$-selection and $\mathrm{ACH}$ mechanism that helps in maximizing the lifetime.

- Evaluate the LCLBM by considering the various parameter such as network lifetime and energy consumption.

This research focuses on achieving the load balancing mechanism and organized in a standard way.

Here, the first section starts with the significance of IoT, WSN, and its application. Further, discuss the problem faced for the implementation and the importance of load balancing. Later, in the same section, 
discuss the motivation and contribution of research work. In the second section, review several existing works for achieving load balancing and their shortcomings. By considering the discussed problem in the literature survey in the third section, develop LCLBM for maximizing through load balancing. The fourth section focuses on evaluating LCLBM through graphical representation by comparing it with the existing protocol.

\section{LITERATURE SURVEY}

In WSN [12], the author has introduced particle swarm optimization (PSO) technique to resolve the load balancing problem and gives routing between CHs. At first, all the given nodes transmit their data to the sink that solves the load balancing, and in-network the sink gives the routing with the help of PSO, which contains the scheme of efficient particle-encoding. Whenever the sensor node ( $\mathrm{SN}$ ) can be assigned via a gateway and the next gateway hop is defined, this information broadcasts the sink on the network. In [13], it introduces the novel algorithm of routing and clustering based on PSO for the WSNs that outperform the introduced algorithm in [12]. The main difference between this work [14] and the same PSO based method is to assume the energy balancing and energy efficiency (EE) simultaneously, where the proposed algorithm only assumes the CHS's EE. In WSNs [14], the author has solved routing and clustering problems by utilizing the PSO. In [13], the main difference between this work and introduced algorithms [14] is used PSO to choose $\mathrm{CHs}$ before the clusters are produced. Anyways, in [12] and [13] gateways that act as the CHs, there is no requirement to choose $\mathrm{CHs}$ to produce the clusters and it only requires assigning SNs to gateways.

In WSNs [15], the author has utilized the genetic algorithm (GA) to solve the routing and clustering algorithm. They assume the gateways' residual energy and distance among corresponding $\mathrm{CH}$ and $\mathrm{SNs}$ for clustering. In addition to gateways residual energy, the trade-off between the number of forwards and transmission distance is assumed for routing. The efficient representation of the chromosome has been efficiently helping to resolve the problems of routing and clustering. In the routing algorithm, the next-hops energy consumptions are not taken into consideration. In paper [16], the author has solved the problems of routing and clustering in the WSNs by utilizing local data about nodes. In this technique, the backbone of directed virtual $\mathrm{CHs}$ is constructed and utilized by the help of $\mathrm{CH}$ to transmit their information to sink. This virtual structure has a root, which is known as the sink. This type of method does not address the load balancing issue. In [17], the author has introduced the algorithm of distributed unequal clustering utilizing fuzzy logic (DUCF) for the WSNs. The DUCF chooses the CHs utilizing the fuzzy method and forms the unequal clusters to balance the power consumption between CHs. In the DUCF, huge numbers of members are assigned to every single $\mathrm{CH}$ based on its number of neighbors, distance, and residual energy to sink.

In [18], have utilized artificial bee colony $(\mathrm{ABC})$ to resolve the problems of routing and clustering in WSNs. The clusters can be produced based on neighborhood data and the energy levels of SNs in the protocol. In $\mathrm{ABC}$, the fitness functions for routing issues are based on the number of hops path and energy efficiency. According to [19], the author has resolved the problem of routing and clustering in WSNs as assuming the problem of a hotspot. The introduced algorithm takes the distance and residual energy from the sink into the account and thus separates the network into not equal size clusters by using the algorithm of multi-objective immune. In paper [20], the author has introduced the protocol for routing and clustering in the WSNs which is based on the type-2 fuzzy logic (T2FL). The prior algorithms have utilized the type-1 fuzzy logic (T1FL) to resolve this issue. T2FL is the sets of fuzzy themselves. In [21], the author has proposed the algorithm of routing and clustering for the WSNs, whereas all nodes are heterogeneous. This method chooses the $\mathrm{CHs}$ in every single round and calculates a smaller number of heterogeneous nodes. The SNs transmit their information to sink with the help of chosen heterogeneous nodes and their $\mathrm{CH}$.

In paper [22], the author has solved the problems of routing and clustering in the WSNs by assuming then as the Pareto optimization problem (multi-objective problem). They have considered the number of CHs and quality of link among the $\mathrm{CHs}$ and cluster members, and the quality of the link is constructed as the routing tree which is taken in the problem of Pareto optimization. In this, the individual encoding method has been designed that permits the joint optimization of routing and clustering issues in the WSNs. In paper [23], the author has defined the problems of routing and clustering in WSNs as the problem of Pareto optimization. This issue is resolved by using the multi-objective particle swarm optimization (MOPSO). In the problem of Pareto optimization, reliability, and EE are described as the function of objective. In the network, they have performed load balancing by revolving the roles of $\mathrm{CH}$ and next hop in each of the iterations. The performance of the network is maximized in comparison to the other algorithm but here load balancing clustering problem has not been addressed. In [24], they have solved the problem of clustering in the WSNs by maximizing the algorithm of Cuckoo search (CS), and the problem of routing is maximized by the algorithm of harmony search. The maximized algorithm of CS discovers an optimal set of CHs between normal SNs. 
In [25], the author has introduced the algorithm of cluster-based routing, which utilizes the convolutional neural network $(\mathrm{CNN})$ and fuzzy rules for WSNs. In the algorithm, there are four types of parameters that remain the same for $\mathrm{CHs}$ energy, the distance among sink and $\mathrm{CHs}$, the nodes, $\mathrm{CH}$, and distance are utilized for the cluster formation. The network can be instructed with the help of fuzzy rules and $\mathrm{CNN}$ for the adjustment weight. Furthermore, they also utilize the approach of fuzzy reasoning for the cluster formation to achieve cluster-based routing. Anyways, they utilize the $\mathrm{CHs}$ degree in the formation of a cluster, and it cannot manage the load of SNs (sensor nodes) between CHs. In [26], introduced the wellknown algorithm as dynamic layered dual-CHs routing based on the optimization of Krill herd $(\mathrm{KH})$ in underwater wireless-sensor networks (UWSNs). The main purpose of this algorithm is to manage the power consumption of CHs. To attain this aim, they utilize the distance between $\mathrm{CH}$ and cluster.

\section{PROPOSED METHOD}

LCLBM comprises several parts such as network model, energy model, ch-sharing model, assistant cluster head- model $(\mathrm{ACH})$ and its functionality, these all are discussed in the following sections.

\subsection{Network model and assumption}

In this sub-section, the network topology of the circular region shaped is designed, here the sink is placed in the center and each layer is in the shape of a square and does not lose any properties. Let us assume that the radius of the network topology is $\mathbb{S}$ and angle $\Theta$, the network topology is parted into the ring shape, where, $e$ is depth. At first all, the nodes are deployed, and it is immobilized, further, each node has the same energy at the initial stage. Each sensor node can scale transmission range and sink has all the information regarding the networks and their parameters, such as, $\mathbb{e}, \mathbb{s}, \mathbb{I}$ and $\Theta$. The data is generated through a uniform distribution. For instance, the data is divided into several fragments in such a way that the traffic flow might be considered as the continuous variable.

\subsection{Energy model}

In this sub-section of the research work, the energy model for the proposed model is introduced. Here, the first-order energy is adopted and meanwhile, the optimized energy model is designed. The transmitter requires energy to run the power amplifier and radio electronics. Receiver transfers energy to the radio electronics and the energy consumption which is required for the $m$ bits to pass to the distance $e$ while transmitting. In (1) presents the energy consumption of the model.

$$
\begin{aligned}
& \mathbb{F}_{\text {Trans }}(m, e)=\mathbb{F}_{\text {Trans }-e l}(m)+\mathbb{F}_{\text {Trans-amp }}(m, e) \\
& \mathbb{F}_{\text {Trans }}(m, e)=\left\{\begin{array}{l}
m * \mathbb{F}_{\text {req }}+m * \xi_{\text {trans }} * e^{2} \text { if } e<e_{0} ; \\
m * \mathbb{F}_{\text {req }}+m * \xi_{\text {amp }} * e^{4} \text { if } e \geq e_{0} ;
\end{array}\right.
\end{aligned}
$$

$\mathbb{F}_{e l}$ is the energy required to run the receiver or transmitter, this depends on the different parameters such as modulation and digital coding $\varepsilon_{\text {trans }}$ and $\varepsilon_{a m p}$ are transmitter and amplifier characteristics respectively. $m$ Indicates data and $e$ indicates distance. Energy consumption required to receive the $m$ data bit as depicted as shown in (2).

$$
\mathbb{F}_{\text {Recv }}(m)=m * \mathbb{F}_{\text {req }}
$$

Threshold value $e_{0}$ is the ratio of transmitter characteristics and amplifier characteristics.

$$
e_{0}=\left(\epsilon_{f s} / \epsilon_{m p}\right)^{-1 / 2}
$$

\subsection{Assistant CH-mechanism modelling}

In this section, Assistant $\mathrm{CH}$-mechanism as well as optimal design for inter-communication such that load balancing is achieved. Further, this section comprises cluster head Sharing, energy minimization, and assistant $\mathrm{CH}$-mechanism.

\subsubsection{Cluster head sharing}

Let us consider the $\mathbb{e}_{o}$ as the distance from the $\mathrm{CH}$ to the sink, further, the energy can be minimized if the following condition (4):

$$
\mathrm{e}_{o}=(2 o-1) \sin 0.5 \Theta(\Theta / \Theta)
$$


If the conditions satisfy, then the distribution is said to be the $\mathrm{CH}$-optimal distribution and (4) needs to be proved. Hence, to prove it assume that the $o^{\text {th }}$ layer area is almost equal to the cluster size and $\mathrm{CH}$ is placed in the location, and the distance between the $\mathrm{CH}$ to sink is $\mathrm{y}$. Let us consider a random node which can be shared randomly at the given coordinate and this given coordinate has the distance of $a$ from $\mathrm{CH}$ and acts as the cluster member. Moreover, if a square distance of all the cluster member to cluster head is smaller than the energy consumption is nominal, hence, the cluster member to the corresponding $\mathrm{CH}$ is given through (5).

$$
\mathbb{b}^{2}=\mathbb{y}^{2}+\mathbb{z}^{2}-2 y z \cos A
$$

In the $(5), \mathbb{Z}$ is the distance between the sink and cluster member and $\mathrm{A}$ is the angle between the Cluster Head and Cluster Member. In cluster distance from the cluster member to the $\mathrm{CH}$ is established through (6).

$$
\sum \mathbb{b}^{2}=\int^{\Theta / 2}-\Theta / 2 \int^{\oplus \mathbb{e}}(o-1) \mathbb{e}\left(\mathbb{y}^{2}+\mathbb{z}^{2}-2 \mathrm{yz} \cos \mathrm{A}\right) d \mathrm{~A} d \mathrm{z}
$$

In formulated (6) can be modified and presented in the (7).

$$
\sum \mathfrak{b}^{2}=3 \Theta\left(3 o^{2}-3 o-1\right) e^{3}+\Theta d y^{2}-2(2 o-1) e^{2} \sin 0.5 \Theta \mathbb{y}
$$

Hence, considering (5), minimize the energy overhead through (6) and (7), further it is observed that $\sum \mathbb{b}^{2}$ can be minimized if coordinates can be drawn through the (8).

$$
\mathbb{Y}=(20-1) \sin 0.5 \Theta y \in \mathbb{y} / \Theta
$$

Thus, once (5) is achieved then optimal $\mathrm{CH}$ can be selected.

\subsection{Energy minimization}

Energy minimization is achieved through the $\mathrm{CH}$-Rotation and $\mathrm{CH}$-selection. This sub-section focus on energy to distance, here it specifically formulate the ratio between the remaining energy of the node and the distance between the node and $\mathrm{CH}$ and this can be denoted as $\mathbb{S}_{\mathbb{F}-\mathbb{e}}$, further (9) presents the mathematical formula for computation.

$$
\mathbb{S}_{\mathbb{F}-\Theta}=\left(e_{t}-C H\right)^{-1} \mathbb{F}_{r e}
$$

As shown in (9), $\mathbb{e}_{t}-\mathrm{CH}$ indicates the distance observed between the node and Cluster Head, $\mathbb{F}_{r e}$ indicates the remaining energy.

\subsection{Assistant cluster head (ACH)}

While developing the cluster, it is observed that $\mathrm{CH}$ observes traffic when compared to its cluster member and this causes the cluster head to consume more energy than its member, hence, the energy consumption must balance. To achieve that the role of these sensor nodes is changed i.e., being $\mathrm{CH}$ and $\mathrm{CM}$. However, while role reversing also it is observed that the energy consumption is more while exchanging the message for their role, to overcome this assistance $\mathrm{CH}$ i.e., $\mathrm{ACH}$ is used. By using the $\mathrm{ACH}$, only $\mathrm{ACH}$ and $\mathrm{CH}$ reverse their role, and this brings energy consumption. In the initial stage, the node which has $\mathbb{S}_{\mathbb{F}-\oplus}$ the larger value is selected and the node's second-highest value $\mathbb{S}_{\mathbb{F}-\mathbb{e}}$ is considered as the $\mathrm{ACH}$, further, the $\mathrm{CH}$ contains the information about the $\mathrm{ACH}$.

\subsubsection{LCLBM based inter-cluster communication model}

This sub-section presents the optimal Inter-cluster communication model, here, consider the node as a player, and each node chooses the nearest node as the next hop for energy minimization. This makes the node for a higher probability to be chosen as the next hop, which has a relatively smaller distance from the several nodes. However, this causes energy exhaustion, and further causes network destruction, to avoid this problem, develop an optimal Inter-cluster communication model. LCLBM model divides into different layers. In the LCLBM strategy model, the model has a set of nodes and amount of data towards the nodes nearest layers, further OICC helps in achieving the equilibrium and equilibrium helps in achieving balanced energy. Furthermore, $\mathrm{CH}$ makes the self-decision for data forwarding to the next hop. Moreover, the LCLBM objective can be given through (10). 
$(\mathbb{Q}, \mathbb{T}, \mathbb{V})$

In the LCLBM model, all nodes at any random layer $\mathrm{n}$ of the given network topology are considered as a set, for instance, if the nodes lie in the $\mathrm{n}^{\text {th }}$ layer is $\mathrm{N}$, then the set of OICC is given as (11).

$$
\mathbb{Q}=\left\{\mathbb{Q}_{j} \backslash 1 \leq j \leq \mathbb{O}\right\}
$$

Further, each node is independent, and it acts without knowing any other node information. In this research strategy of each node is denoted in (12).

$$
\mathbb{T}=\left(\mathbb{t}_{1}, \mathbb{t}_{2}, \ldots \ldots, \mathbb{T}_{\mathbb{Q}}\right)
$$

The node strategy can be formulated as shown in (13).

$$
\mathbb{T}_{j}=\mathbb{E}_{j k}
$$

Where $\mathbb{E}_{j k}$ indicates the data from node $j$ to node $k$. Similarly, the node capacity can be given as (14),

$$
\mathbb{D}_{k}=\mathbb{F}_{k}\left(\mathbb{f}_{r x}\right)^{-1}
$$

In (14), $\mathbb{F}_{k}$ is the energy cost to receive the data. Further, to balance the load, load balancing is given in (15), where $\mathbb{e}_{\min }$ indicates the minimum distance between the neighboring layers and $\mathbb{e}_{j k}$ is the distance from the node $j$ to node $k$.

$$
\Theta_{j}=\mathbb{e}_{\min }\left(\Theta_{j k}\right)^{-1}
$$

The utility function can be formulated through (15).

$$
\mathbb{V}_{j}\left(\mathbb{t}_{j}, \mathbb{t}_{-j}\right)=\left(\mathbb{D}_{k}-\sum_{j=1}^{\Phi} \mathbb{E}_{j k}\right) \mathbb{E}_{j k}^{\theta}
$$

In above equation, $\mathbb{t}_{-j}$ is the strategy set for the node. Further, from equation 16 transmitter $j$ and receiver $k$, both are considered with parameters defined in (15) and (16), this helps in achieving the load balance. Let $\mathbb{V}_{j}\left(\mathbb{t}_{j}, \mathbb{t}_{-j}\right)$ be the utility of the function then establish the load balancing phenomena (LBP). Moreover, to achieve that the following equation needs to be established, where, the utility function $\mathbb{V}_{j}\left(\mathbb{E}_{i}, \mathbb{E}_{-j k}\right)$ can be maximum.

$$
\mathbb{E}_{j k}^{*}=\Theta_{j} \cdot \mathbb{D}_{k}\left(1+\sum_{j=1}^{\mathbb{Q}} \Theta_{j}\right)^{-1}
$$

To establish the maximum, first, consider $\mathbb{V}_{j}\left(\mathbb{E}_{j}, \mathbb{E}_{-j k}\right)$ as $\mathbb{V}_{j}($.$) , the value of \mathbb{V}_{j}($.$) is maximum$ only if $\frac{\partial \mathbb{V}_{j}(.)}{\partial \mathbb{E}_{j k}}$ is null and this can be formulated in (18).

$$
\frac{\partial \mathbb{V}_{j}(.)}{\partial \mathbb{E}_{j k}}=\Theta^{j} \mathbb{E}_{j k}^{\Theta j-1}\left(\mathbb{D}_{k}-\sum_{i=1}^{o} \mathbb{E}_{i j}\right)-\mathbb{E}_{i j}^{\Theta i}=0,
$$

Using (18), achieve (19), here, $i$ value can vary from 1 to $\mathbb{O}$, and after various iteration, it achieves the optimized LBP i.e.,in (20).

$$
\mathbb{E}_{i j}=\Theta_{j} \mathbb{E}_{k}-\Theta_{j k} \sum_{j=1}^{o} \mathbb{E}_{j k}
$$

Further, using (20) in formulated (19), develop the load balanced phenomena (LBP) which is depicted in (21).

$$
\begin{aligned}
& \sum_{j=1}^{o} \mathbb{E}_{j k}=\mathbb{D}_{k}\left(\Theta_{1}+\Theta_{2}+\cdots+\Theta_{\mathbb{Q}}\right)\left(1+\Theta_{1}+\Theta_{2}+\cdots+\Theta_{\mathbb{O}}\right)^{-1} \\
& \mathbb{E}_{j k}=\Theta_{i} \cdot \mathbb{D}_{k}\left(1+\sum_{j=1}^{N} \Theta_{j}\right)^{-1}
\end{aligned}
$$




\subsection{LCLBM functionality}

This section describes the energy-efficient mechanism, and it is three-stage that can be named as $\mathrm{CH}$ selection, data acquisition, and assistant $\mathrm{CH}$-selection.

\subsubsection{LCLBM based CH-selection}

- Step 1: Here the base station identifies the efficient distribution by the network topology parameter named as $\Theta$, e, $\mathbb{S}$ and $\mathbb{I}$.

- Step 2: Once the optimized cluster head sharing is done, then the node computes the optimized distance from the cluster head and the remaining energy is also computed.

- Step 3: Once the optimal distribution of $e_{o}$; further sensor node computes the corresponding distance $e_{\mathbb{t}-C H}$ later it obtains $\mathbb{S}_{F-e}$ from (9).

- Step 4: Further, the sensor node broadcasts the CH-selection and contains the information of o, ID, $\mathbb{S}_{F-e}$, once, the sensor node receives the broadcasting message it compares the ratio computed, if the ratio of that particular node is higher than it creates $\mathrm{CH}_{-}$alert by recommending itself as cluster head. Hence, the node that has the highest ratio is selected as the cluster head.

- Step 5: Similarly, cluster members select the proper cluster Head, this is carried through a invite message, which is broadcasted by the cluster head, this invite message contains the various information such as layer number, sender ID, and energy minimization phenomena (EMP).

- Step 6: Further ACH is chosen through the sorting of all the computed ratio, where the highest is chosen as custer head and the second highest is chosen as the ACH.

\subsection{Data-CPT (collecting, processing and transmission) phase}

This phase can be divided into two distinctive part i.e., inter and intra.

\subsubsection{Inter data- CPT}

- Step 1: Once the clusters are formed, $\mathrm{CH}$ divides the time slot following cluster member and simultaneously it allocates the time top cluster member.

- Step 2: Hence, in the given slotted time, the node performs the CPT whereas other nodes are kept in REST mode.

\subsubsection{Intra data-CPT}

- Step 1: After cluster formation and $\mathrm{CH}$ has been selected then the arbitrary layer computes the capacity $\mathbb{D}_{k}$ that is given in $(20)$.

- Step 2: It notifies all cluster head which lies in the $\mathrm{n}^{\text {th }}$ layer of $\mathbb{E}_{k}$ through broadcasting.

- Step 3: When all the $\mathrm{CH}$ gets the $\mathbb{E}_{k}$ value then the distance is calculated to the respective cluster head. Further, balancing factor $\Theta_{j}$ is achieved through broadcasting.

- Step 4: Once the cluster head has full information of balancing factors, then it tries to establish the optimal data to nodes at the nth layer, later the data that lies in an upstream layer is regulated following $\mathbb{E}_{j k}^{*}$.

\subsection{Assistant CH-protocol functionality steps}

To reduce the energy overhead and balance use Assistant Cluster Head, this mechanism is carried out in the following steps:

- Step 1: After each round, the present $\mathrm{CH}$ is compared with the ratio of assistant $\mathrm{CH}$; in case if the ratio of present $\mathrm{CH}$ is less then it sends a message.

- Step 2: If $\mathrm{CH}$ assistant $\mathrm{CH}$ decides to be $\mathrm{CH}$, then it sends a message and waits for the acknowledgment from the cluster member.

- Step 3: Once the acknowledgment is received, members keep track of information about the next ACH.

- Step 4: If the ratio computed by ACH is lower than the present cluster head then the discard is alerted, and cluster head continues to be $\mathrm{CH}$. Hence, this sub mechanism tends to achieve load balancing. Once the mechanism is developed, it evaluates the LCLBM-mechanism in the next section.

\section{PERFORMANCE EVALUATION}

Wireless sensor networks has a variety of applications, it is continuously rising despite various constraints such as storage, communication range, processing capacity, and energy. The primary issue is its lifetime, and this occurs due to more energy consumption. This issue can be tackled through balancing the load and it helps in energy consumption. In this section, evaluate PS-LCLBM and the evaluation is carried 
out using system parameter with windows 10-OS (Operating System) with Quad-Core processor (64 bit) and the system is packed with 2GB NVIDIA CUDA and 16 GB of RAM. The simulation is carried out using the sensoria simulator [27] and PS-LCLBM is programmed using the C\# language. Table 1 presents further details about the system parameter.

Table 1. Simulation parameter

\begin{tabular}{cc}
\hline Network Parameter & Value \\
\hline Network Size & $75 \mathrm{~m} * 75 \mathrm{~m}$ \\
Amplification energy (Emp) & $100 \mathrm{pJ} / \mathrm{bit} / \mathrm{m} 2$ \\
Number of sensor nodes & $500,1000,1500 \mathrm{and} 2000$ \\
Data packet processing delay & $0.1 \mathrm{~ms}$ \\
Number of Base stations & 2 \\
The initial energy of sensor nodes & $0.2 \mathrm{~J}$ \\
Radio energy dissipation & $50 \mathrm{NJ} / \mathrm{bit}$ \\
Idle energy consumption (Elec) & $50 \mathrm{NJ} / \mathrm{bit}$ \\
Data packets length & $2000 \mathrm{bits}$ \\
Bandwidth & $5000 \mathrm{bit} / \mathrm{s}$ \\
Transmission speed & $100 \mathrm{bit} / \mathrm{s}$ \\
\hline
\end{tabular}

\subsection{Comparative analysis}

This section performs the comparative analysis between the PS-LCLBM and the existing model by considering the various parameter such as network lifetime, One-way delay, active nodes, and communication overhead.

\subsubsection{Network lifetime}

Energy efficiency is always used as the criterion with the network lifetime; due to the limited amount of energy given to each sensor. Hence, in this section, the network lifetime comparison is considered through varying nodes. In Figures 2-5, lifetime comparison has been carried for 500 nodes, 1000 nodes, 1500 nodes, and 2000 nodes, respectively. Here, it is observed that in the case of an existing model the network lifetime is marginally less in comparison with an existing model. Hence, the observation can be made that the proposed model depreciates the energy consumption and thus increases the network lifetime.

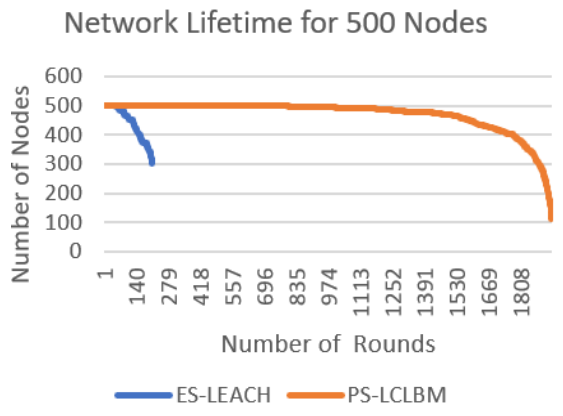

Figure 2. Network lifetime for 500 node

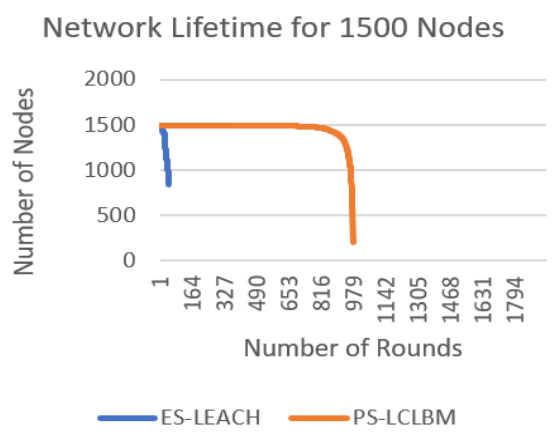

Figure 4. Network lifetime for 1500 node

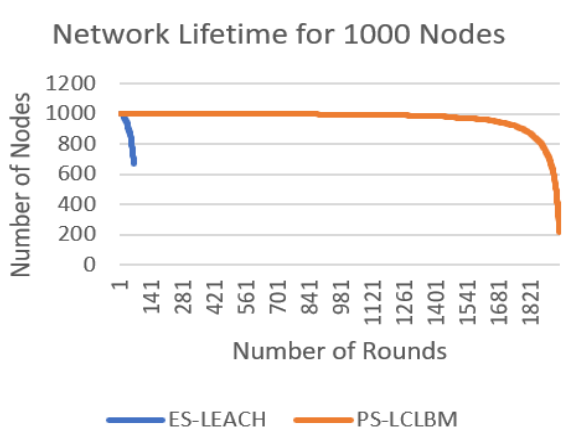

Figure 3. Network lifetime for 1000 nodes

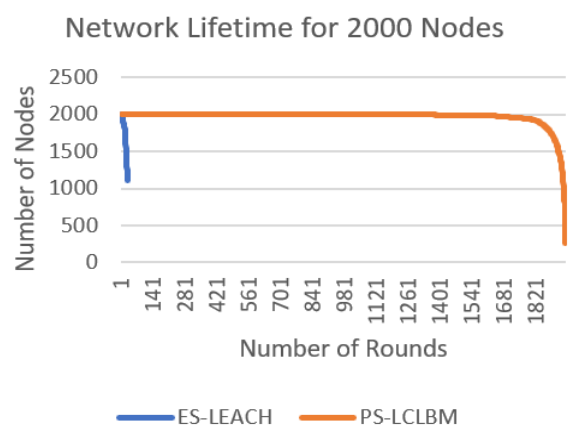

Figure 5. Network lifetime for 2000 nodes 


\subsubsection{Energy consumption}

Energy consumption is one of the key parameters for evaluation of the load balancing mechanism and the sensor nodes are battery-based and possesses a limited amount of energy. Hence, energy consumption becomes essential parameter. Here, the existing model is compared with the proposed model LCLBM by varying the sensor number of nodes as 500 nodes, 1000 nodes, 1500 nodes, and 2000 nodes. Further, the comparison is tabulated in Table 2.

Table 2. Simulation parameter

\begin{tabular}{ccc}
\hline No. of sensor nodes & ES-LEAH (energy in mj) & PS-LCLBM (energy in mj) \\
\hline 500 & 30.53496296 & 24.85570736 \\
1000 & 64.05439272 & 47.70517578 \\
1500 & 86.7461309 & 75.57840514 \\
2000 & 119.4031467 & 99.62671092 \\
\hline
\end{tabular}

\subsubsection{Overhead}

This section proves the efficiency of PS-LCLBM by comparing the communication overhead and it is defined as the portion of time spent on communicating with the node. Higher communication overhead results in more energy consumption and Figure 6 average communication overhead are compared. In case of 500 nodes, communication overhead (CO) is 0.1508 whereas CO of LCLBM is 0.025. Similarly, for 1000 nodes, 1500 nodes and 2000 nodes communication overhead for the existing mechanism is $2.14,3.72$, and 5.12 also CO for PS- LCLBM is $0.15,0.17$, and 0.20 , respectively. Through the comparative analysis, it is observed that communication overhead increase exponentially with the increase of the number of nodes for the existing model and CO increases linearly for LCLBM.

\subsubsection{The active number of nodes}

This is another performance metric for evaluation of PS-LCLBM; In load balancing the energy is the very important part as the energy must be shared efficiently and the load should be balanced in such a way that energy can be utilized with a greater number of active nodes i.e., nodes that are performing the data transmission. Hence, a higher number of active nodes indicates less load on the node, and energy can be reduced. Further, the lifetime of the network is also increased, graphical comparison has been presented in Figure 7.

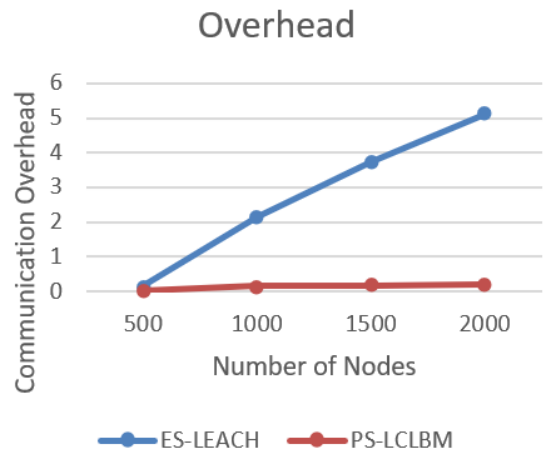

Figure 6. Communication overhead comparison

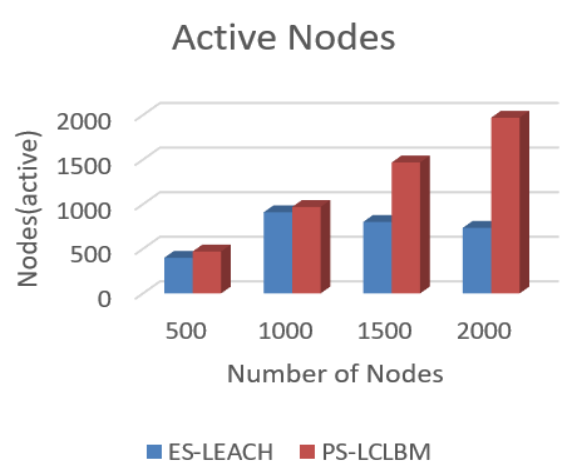

Figure 7. Active number of node comparison

\section{CONCLUSION}

This research work proposes LCLBM for balancing the load on the network in IoT. Here, a specific network is designed to establish the ideal environment for load balancing then later with the help of the ACH, the loads are balanced to maximize the lifetime of the network. LCLBM is evaluated by comparing the important performance metrics with the existing model, such as, network lifetime, energy consumption, network length, and overhead. In terms of energy consumption for 500 nodes, 1000 nodes, 1500 nodes, and 2000 nodes the proposed model achieves better results as it consumes $18.60 \%, 25.52 \%, 12.87 \%$, and $16.56 \%$ respectively less energy than the existing model. Similarly, considering different parameters such as one-way delay communication overhead, nodes currently performing, network length, and communication overhead, a comparative analysis is carried out and through the analysis, it is observed that the proposed model not only 
outperforms the existing model but also provides the way for further research in optimizing the load balancing. However, considering the importance and popularity of WSN, still several constraints such as quality of service considering the demand for bandwidth, data processing, and compressing technique needs to be considered in future research.

\section{REFERENCES}

[1] G. Bedi, G. K. Venayagamoorthy, R. Singh, R. R. Brooks and K. Wang, "Review of Internet of Things (IoT) in Electric Power and Energy Systems," in IEEE Internet of Things Journal, vol. 5, no. 2, pp. 847-870, April 2018, doi: 10.1109/JIOT.2018.2802704.

[2] O. Elijah, T. A. Rahman, I. Orikumhi, C. Y. Leow and M. N. Hindia, "An Overview of Internet of Things (IoT) and Data Analytics in Agriculture: Benefits and Challenges," in IEEE Internet of Things Journal, vol. 5, no. 5, pp. 3758-3773, Oct. 2018, doi: 10.1109/JIOT.2018.2844296.

[3] H. Kim and S. Han, "An Efficient Sensor Deployment Scheme for Large-Scale Wireless Sensor Networks," in IEEE Communications Letters, vol. 19, no. 1, pp. 98-101, Jan. 2015, doi: 10.1109/LCOMM.2014.2372015.

[4] L. Xu, R. Collier and G. M. P. O'Hare, "A Survey of Clustering Techniques in WSNs and Consideration of the Challenges of Applying Such to 5G IoT Scenarios," in IEEE Internet of Things Journal, vol. 4, no. 5, pp. 1229-1249, Oct. 2017, doi: 10.1109/JIOT.2017.2726014.

[5] J. N. Al-Karaki and A. Gawanmeh, "The Optimal Deployment, Coverage, and Connectivity Problems in Wireless Sensor Networks: Revisited," in IEEE Access, vol. 5, pp. 18051-18065, 2017, doi: 10.1109/ACCESS.2017.2740382.

[6] Y. Liao, H. Qi and W. Li, "Load-Balanced Clustering Algorithm With Distributed Self-Organization for Wireless Sensor Networks," in IEEE Sensors Journal, vol. 13, no. 5, pp. 1498-1506, May 2013, doi: 10.1109/JSEN.2012.2227704.

[7] F. Wang, S. Wu, K. Wang and X. Hu, "Energy-Efficient Clustering Using Correlation and Random Update Based on Data Change Rate for Wireless Sensor Networks," in IEEE Sensors Journal, vol. 16, no. 13, pp. 5471-5480, July1, 2016, doi: 10.1109/JSEN.2016.2561283.

[8] S. Kumar, "Compartmental Modeling of Opportunistic Signals for Energy Efficient Optimal Clustering in WSN," in IEEE Communications Letters, vol. 22, no. 1, pp. 173-176, Jan. 2018, doi: 10.1109/LCOMM.2017.2763948.

[9] M. M. Alam, M. A. Razzaque, M. Mamun-Or-Rashid and C. S. Hong, "Energy-aware QoS provisioning for wireless sensor networks: Analysis and protocol," in Journal of Communications and Networks, vol. 11, no. 4, pp. 390-405, Aug. 2009, doi: 10.1109/JCN.2009.6391353.

[10] X. Liu and P. Zhang, "Data Drainage: A Novel Load Balancing Strategy for Wireless Sensor Networks," in IEEE Communications Letters, vol. 22, no. 1, pp. 125-128, Jan. 2018, doi: 10.1109/LCOMM.2017.2751601.

[11] S. Hu and G. Li, "Fault-Tolerant Clustering Topology Evolution Mechanism of Wireless Sensor Networks," in IEEE Access, vol. 6, pp. 28085-28096, 2018, doi: 10.1109/ACCESS.2018.2841963.

[12] Kuila, P. and Jana, P. K., "Energy-efficient clustering and routing algorithms for wireless sensor networks: particle swarm optimization approach," Engineering Applications of Artificial Intelligence, vol. 33, pp. 127-140, 2014, doi: 10.1016/j.engappai.2014.04.009.

[13] Azharuddin, M. and Jana, P. K., "PSO-based approach for energy-efficient and energy-balanced routing and clustering in wireless sensor networks," Soft Computing, vol. 21, no. 22, pp. 6825-6839, 2017, doi: 10.1007/s00500-016-2234-7.

[14] R. S. Y. Elhabyan and M. C. E. Yagoup, "Two-tier particle swarm optimization protocol for clustering and routing in the wireless sensor network," Applications, vol. 52, pp. 116-128, 2015, doi: 10.1016/j.jnca.2015.02.004.

[15] Gupta, S. K. and Jana, P. K., "Energy-efficient clustering and routing algorithms for wireless sensor networks: GA based approach," Wireless Personal Communications, vol. 83, no. 3, pp. 2403-2423, 2015, doi: 10.1007/s11277-015-2535-7.

[16] T. Amgoth and P. K. Jana, "An energy-aware routing algorithm for wireless sensor networks," Computers \& Electrical Engineering, vol. 41, pp. 357-367, 2015, doi: 10.1016/j.compeleceng.2014.07.010.

[17] B. Baranidharan and B. Santhi, "DUCF: distributed load balancing Unequal Clustering in wireless sensor networks using Fuzzy approach,” Applied Soft Computing, vol. 40, pp. 495-506, 2016, doi: 10.1016/j.asoc.2015.11.044.

[18] A. A. A. Ari, B. O. Yenke, N. Labraoui, and D. Irepran, "A power-efficient cluster-based routing algorithm for wireless sensor networks: honeybees swarm intelligence-based approach," Journal of Network and Computer Applications, vol. 69, pp. 77-97, 2016, doi: 10.1016/j.jnca.2016.04.020.

[19] N. Sabor, M. Abo-zahhad, S. Sasaki, and S. M. Ahmed, "An unequal multi-hop balanced immune clustering protocol for wireless sensor networks," Applied Soft Computing, vol. 43, pp. 372-389, doi: 10.1016/j.asoc.2016.02.016.

[20] P. Nayak and B. Vathasavai, "Energy Efficient Clustering Algorithm for Multi-Hop Wireless Sensor Network Using Type-2 Fuzzy Logic," in IEEE Sensors Journal, vol. 17, no. 14, pp. 4492-4499, 15 July15, 2017, doi: 10.1109/JSEN.2017.2711432.

[21] L. Chunli, B. Jingpan, G. Jinguang, Y. Xin, and L. Youlong, "Clustering routing based on mixed-integer programming for heterogeneous wireless sensor networks," Ad Hoc Networks, vol. 72, pp. 81-90, 2018, doi: 10.1016/j.adhoc.2018.02.001. 
[22] R. Elhabyan, W. Shi, and M. St-Hilaire, "A Pareto optimization-based approach to clustering and routing in Wireless Sensor Networks," Journal of Network and Computer Applications, vol. 114, pp. 57-69, 2018, doi: 10.1016/j.jnca.2018.04.005.

[23] S. Randhawa and S. Jain, "MLBC: multi-objective Load Balancing Clustering technique in Wireless Sensor Networks," Applied Soft Computing, vol. 74, pp. 66-89, 2019, doi: 10.1016/j.asoc.2018.10.002.

[24] G. P. Gupta and S. Jha, "Integrated clustering and routing protocol for wireless sensor networks using Cuckoo and Harmony Search based metaheuristic techniques," Engineering Applications of Artificial Intelligence, vol. 68, pp. 101-109, 2018, doi: 10.1016/j.engappai.2017.11.003.

[25] K. Thangaramya, K. Kulothungan, R. Logambigai, M. Selvi, S. Ganapathy, A. Kannan, "Energy-aware cluster and neuro-fuzzy based routing algorithm for wireless sensor networks in IoT," Computer Networks, vol. 151, pp. 211-223, 2019, doi: 10.1016/j.comnet.2019.01.024.

[26] P. Jiang, Y. Feng, F. Wu, S. Yu, and H. Yu, "Dynamic layered dual-cluster heads routing algorithm based on krill herd optimization in UWSNs," Sensors, vol. 16, no. 9, p. 1379, 2016, doi: 10.3390/s16091379.

[27] J. N. Al-Karaki and G. A. Al-Mashaqbeh, "SENSORIA: A New Simulation Platform for Wireless Sensor Networks," 2007 International Conference on Sensor Technologies and Applications (SENSORCOMM 2007), Valencia, Spain, 2007, pp. 424-429, doi: 10.1109/SENSORCOMM.2007.4394958. 